Recombination Dynamics in

PbS Nanocrystal Quantum Dot

Solar Cells Studied through Drift-

Diffusion Simulations

Journal Article

Author(s):

Lin, Weyde (1); Yazdani, Nuri (1); Yarema, Olesya (1); Yarema, Maksym (1); Liu, Mengxia; Sargent, Edward H.; Kirchartz, Thomas; Wood, Vanessa (1)

Publication date:

2021-11-23

Permanent link:

https://doi.org/10.3929/ethz-b-000515464

Rights / license:

Creative Commons Attribution-NonCommercial-NoDerivatives 4.0 International

Originally published in:

ACS Applied Electronic Materials 3(11), https://doi.org/10.1021/acsaelm.1c00787 


\title{
Recombination Dynamics in PbS Nanocrystal Quantum Dot Solar Cells Studied through Drift-Diffusion Simulations
}

\author{
Weyde M. M. Lin, Nuri Yazdani, Olesya Yarema, Maksym Yarema, Mengxia Liu, Edward H. Sargent, \\ Thomas Kirchartz, and Vanessa Wood*
}

Cite This: ACS Appl. Electron. Mater. 2021, 3, 4977-4989

Read Online

\section{ACCESS | Lill Metrics \& More | 回 Article Recommendations ｜（） Supporting Information}

ABSTRACT: The significant performance increase in nanocrystal (NC)-based solar cells over the last decade is very encouraging. However, many of these gains have been achieved by trial-anderror optimization, and a systematic understanding of what limits the device performance is lacking. In parallel, experimental and computational techniques provide increasing insights into the electronic properties of individual NCs and their assemblies in thin films. Here, we utilize these insights to parameterize drift-diffusion simulations of $\mathrm{PbS} \mathrm{NC}$ solar cells, which enable us to track the distribution of charge carriers in the device and quantify recombination dynamics, which limit the device performance. We simulate both Schottky- and heterojunction-type devices and,
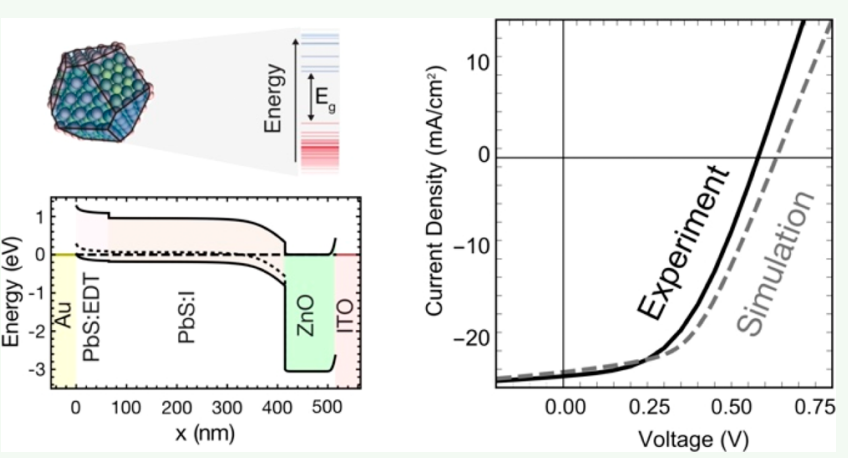
through temperature-dependent measurements in the light and dark, experimentally validate the appropriateness of the parameterization. The results reveal that Schottky-type devices are limited by surface recombination between the $\mathrm{PbS}$ and aluminum contact, while heterojunction devices are currently limited by NC dopants and electronic defects in the $\mathrm{PbS}$ layer. The simulations highlight a number of opportunities for further performance enhancement, including the reduction of dopants in the nanocrystal active layer, the control over doping and electronic structure in electron- and hole-blocking layers (e.g., $\mathrm{ZnO}$ ), and the optimization of the interfaces to improve the band alignment and reduce surface recombination. For example, reduction in the percentage of p-type NCs from the current $1-0.01 \%$ in the heterojunction device can lead to a $25 \%$ percent increase in the power conversion efficiency.

KEYWORDS: nanocrystal, quantum dot, lead sulfide, solar cell, simulation, device architecture

\section{INTRODUCTION}

Nanocrystal (NC)-based solar cells are promising candidates for next-generation photovoltaics, offering tunable band gaps ${ }^{1}$ and potentially low-cost manufacturing via solution processing. ${ }^{2}$ There has been significant improvement in the device performance over the last 15 years, with initial solar cells from 2005 showing a just few percent power conversion efficiency ${ }^{3}$ to the most recent devices offering $15 \%{ }^{4}$ Lead sulfide (PbS) NC-based solar cells have achieved efficiencies of $12.4 \%$ and are the focus of our work here since they have been a driver in the development of $\mathrm{NC}$ solar cell understanding and technology.

Changes to the active materials and the device architecture have increased the device performance, with improvements often linked to a reduction in non-radiative recombination. From early on, it was apparent that non-radiative recombination through electronic trap states in the NC thin films was limiting the device performance, ${ }^{6}$ and significant effort has been directed toward the reduction of trap states through optimization of the NC passivation. ${ }^{7-12}$ In addition, modern device designs employ layered ${ }^{13,14}$ or doping-graded ${ }^{15,16} \mathrm{NC}$ thin films to improve charge separation and extraction, thereby mitigating the impact of trap-assisted recombination.

Further improvement in the device performance can be accelerated with in depth knowledge of the recombination processes occurring in the solar cells under operating conditions. Fitting analytical models to experimental data provides important insights. ${ }^{17,18}$ For example, identifying trapassisted Shockley-Read-Hall (SRH) recombination as the dominant recombination process in $\mathrm{PbS} \mathrm{NC}$ solar cells, and determining the impact of the $\mathrm{NC}$ band gaps and mobilities on recombination dynamics, was possible through fitting to temperature-dependent current-voltage $(I-V)$ measurements. ${ }^{19,20}$ While such approaches can provide insights into the dominant recombination mechanism, they only offer

Received: August 27, 2021

Accepted: October 13, 2021

Published: November 2, 2021 


\section{Schottky}
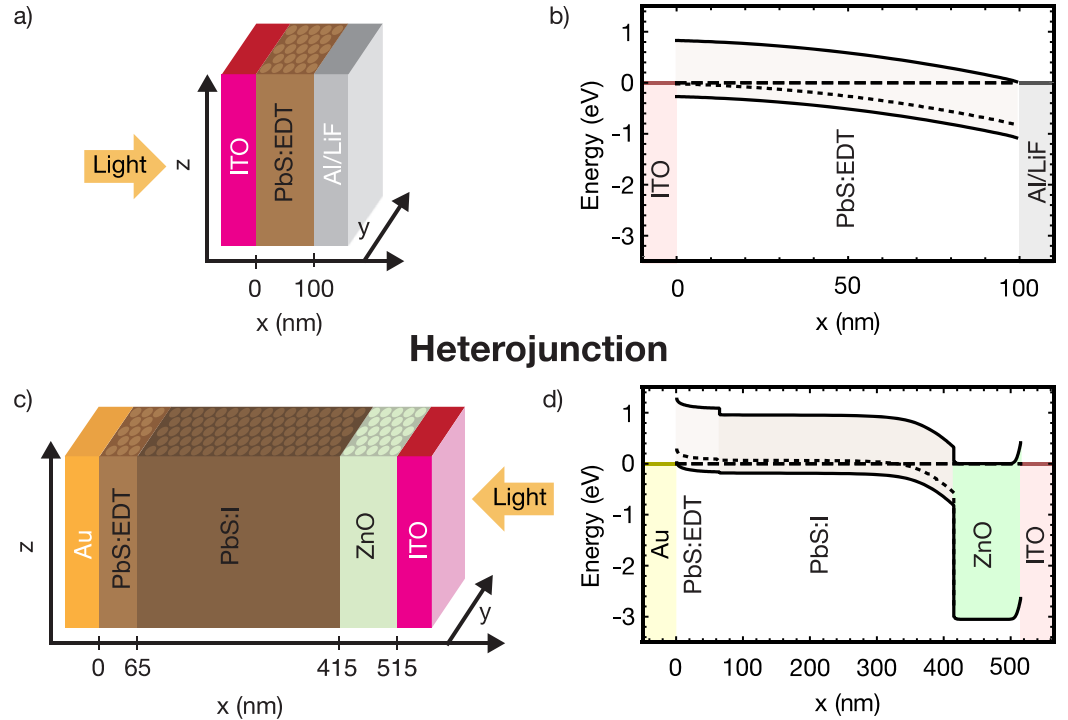

Figure 1. Schematics showing (a) architecture and (b) energy band diagram (at 0 V, no illumination) of the "Schottky" metal-semiconductormetal device, and (c) architecture and (d) energy band diagram (at $0 \mathrm{~V}$, no illumination) of the heterojunction device investigated experimentally and with simulations.

Table 1. Selected Input Parameters ${ }^{a}$

\begin{tabular}{|c|c|c|c|c|}
\hline name & variable & value & unit & source \\
\hline \multicolumn{5}{|c|}{$\mathrm{PbS}$} \\
\hline band gap & $E_{\mathrm{g}}$ & & $\mathrm{eV}$ & Bozyigit et al. $^{19}$ \\
\hline Schottky: PbS:EDT & & 1.10 & & \\
\hline heterojunction: $\mathrm{PbS}: \mathrm{EDT}$ & & 1.25 & & \\
\hline heterojunction: PbS:I & & 1.14 & & \\
\hline electron affinity & $E_{\mathrm{C}}$ & & $\mathrm{eV}$ & see ref 22 \\
\hline PbS:EDT & & 3.79 & & \\
\hline PbS:I & & 3.92 & & \\
\hline electron mobilities at $300 \mathrm{~K}$ & $\mu_{\mathrm{e}}$ & 1.65 & $\mathrm{~cm}^{2} / \mathrm{V} \mathrm{s}$ & see text and ref 22 \\
\hline hole mobilities at $300 \mathrm{~K}$ & $\mu_{\mathrm{h}}$ & 0.18 & $\mathrm{~cm}^{2} / \mathrm{V} \mathrm{s}$ & see text and ref 22 \\
\hline shallow doping density & & 0 & $\mathrm{~cm}^{-3}$ & see text and ref 22 \\
\hline defect type & & acceptor & & see text and ref 22 \\
\hline deep doping density & $N_{\mathrm{A}}$ & $10^{17^{1}}$ & $\mathrm{~cm}^{-3}$ & see text and ref 22 \\
\hline capture cross section e/h & $\sigma_{\mathrm{e}} / \sigma_{\mathrm{h}}$ & $10-{ }^{13}$ & $\mathrm{~cm}^{2}$ & see text and ref 22 \\
\hline energy level with respect to $E_{\mathrm{V}}$ & $E_{\mathrm{T}, \mathrm{A}}$ & 0.25 & $\mathrm{eV}$ & see text and ref 22 \\
\hline name & variable & value & unit & source \\
\hline \multicolumn{5}{|c|}{$\mathrm{ZnO}$} \\
\hline band gap & $E_{\mathrm{g}}$ & 3.05 & $\mathrm{eV}$ & see Peng et al. ${ }^{25}$ \\
\hline electron affinity & $E_{\mathrm{C}}$ & 4.19 & $\mathrm{eV}$ & see text \\
\hline CB effective density of state & $N_{\mathrm{C}}$ & $3.14 \times 10^{18}$ & $\mathrm{~cm}^{-3}$ & see text \\
\hline VB effective density of state & $N_{\mathrm{v}}$ & $2.51 \times 10^{19}$ & $\mathrm{~cm}^{-3}$ & see text \\
\hline electron mobilities & $\mu_{\mathrm{e}}$ & 0.5 & $\mathrm{~cm}^{2} / \mathrm{V} \mathrm{s}$ & see text \\
\hline hole mobilities & $\mu_{\mathrm{h}}$ & 0.15 & $\mathrm{~cm}^{2} / \mathrm{V} \mathrm{s}$ & see text \\
\hline shallow donor doping density & $N_{\mathrm{D}}$ & $3 \times 10^{18}$ & $\mathrm{~cm}^{-3}$ & see text \\
\hline defects & & none & & see text \\
\hline
\end{tabular}

limited insights into where recombination occurs spatially within the device.

Numerical drift diffusion simulations can be used to gain further insights into the spatial distribution of charge and the recombination mechanisms under different operating conditions. One challenge in drift-diffusion simulations of solar cells is that they require a large number of input parameters (on the order of hundreds), ${ }^{21}$ which leads to the risk of overfitting. Indeed, different combinations of parameters could lead to satisfactory agreement to measurements (e.g., the same short-circuit current could be fit using a high absorptivity and a high recombination or a low absorptivity and low recombination). In the solar cell literature, this challenge of overfitting is often addressed by designing experiments to extract or performing calculations to determine the parameters independently, therefore only fitting a small select number of 
a)

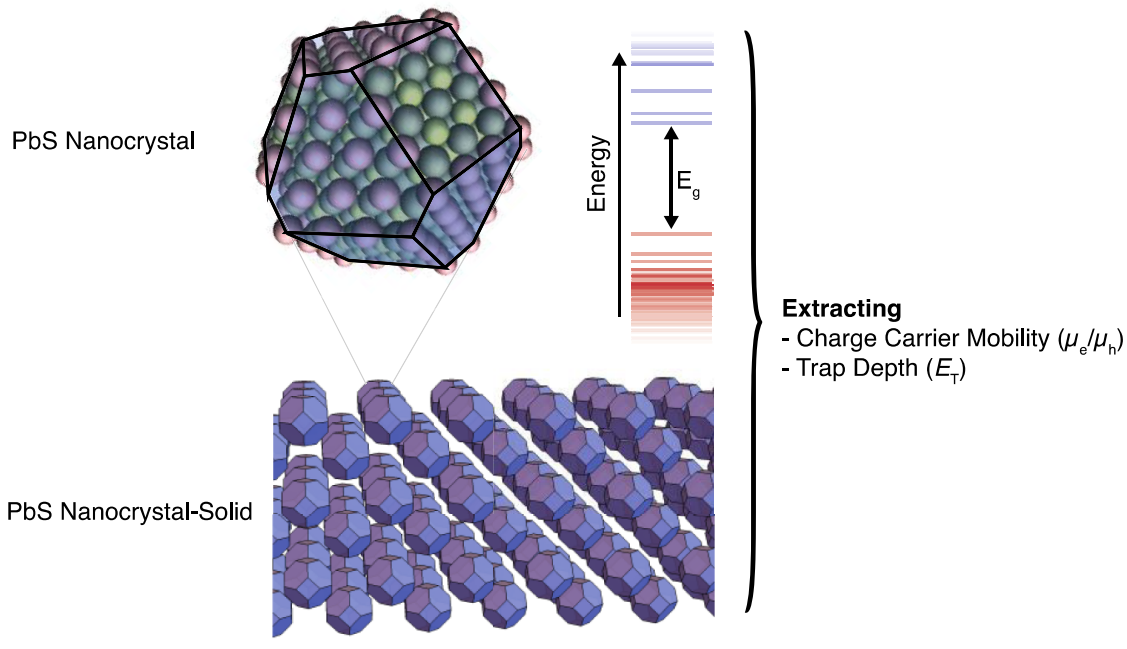

b)

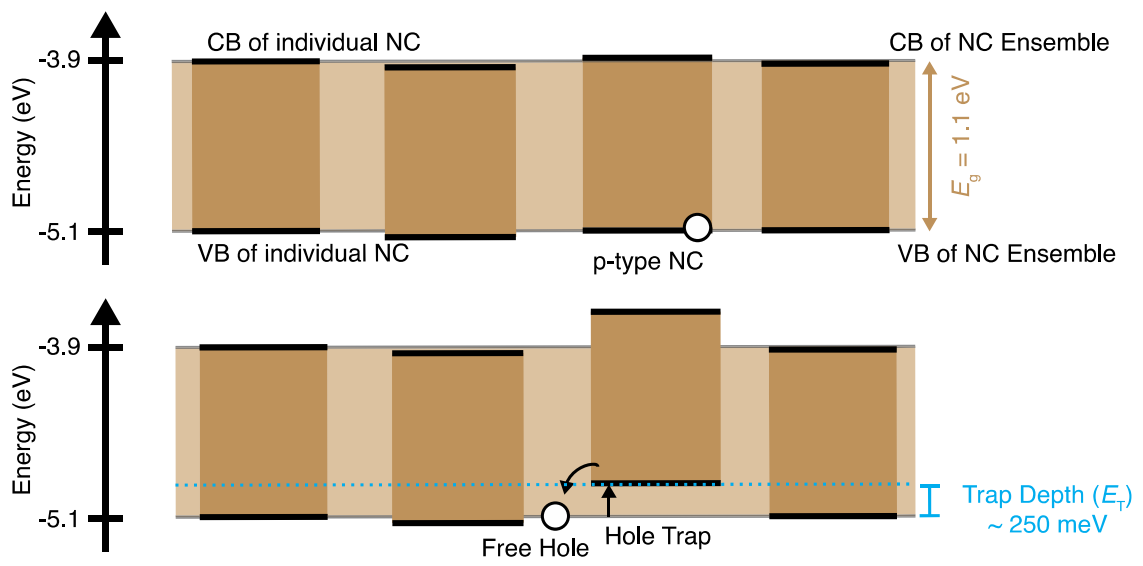

c)
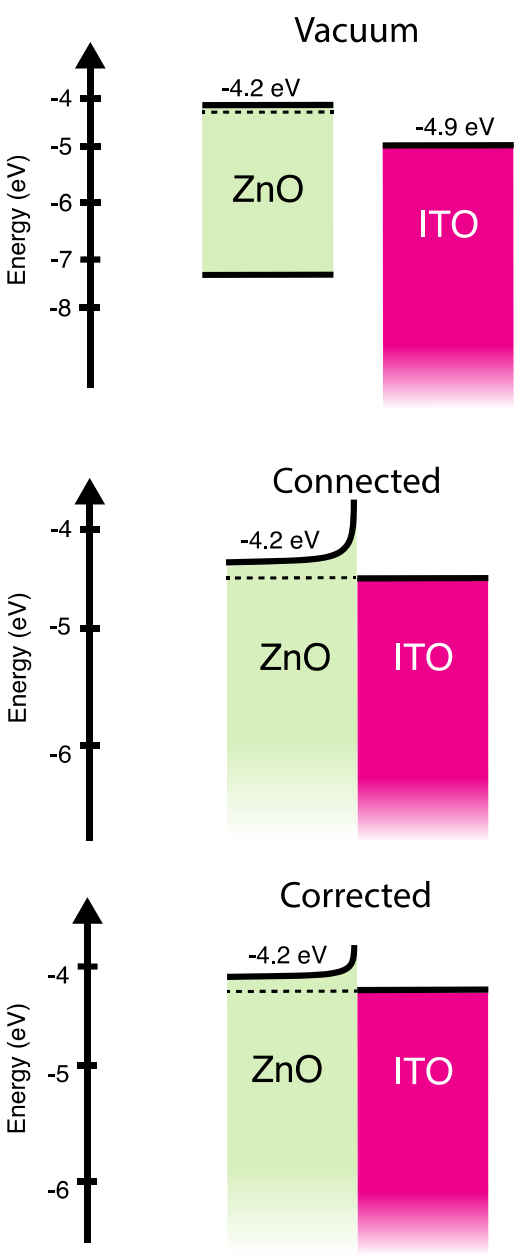

Figure 2. (a) Ensembled individual PbS NCs form the NC solid, (b) trap formation in a NC-film, and (c) ZnO-ITO interface formation.

parameters. We have shown that we are now at a point in our understanding of $\mathrm{NC}$ materials that it is possible to take systemically measured and calculated parameters to fully simulate NC solar cells at the device level. ${ }^{22}$

Here, we perform numerical drift-diffusion simulations of metal-semiconductor-metal and heterojunction $\mathrm{PbS} \mathrm{NC}$ based solar cells to understand the band structure of these devices, the charge distributions, and recombination dynamics. We carry out a systematic parameterization of the devices, and agreement between simulated and experimental temperaturedependent current-voltage $(I-V)$ measurements in the dark, $I-V$ measurements under 1 sun illumination, and temperatureand illumination-dependent open-circuit voltage $\left(V_{\mathrm{OC}}\right)$ measurements confirm the appropriateness of the parameterization. The simulations enable us to gain detailed insights into what types of recombination occur and where it occurs in the different device architectures. Finally, we use the simulations to highlight how device performance can be further improved by judicious selection and optimization of blocking contacts and the dopant and trap state density in the NC layer.

\section{EXPERIMENT AND SIMULATION}

Device Fabrication. We experimentally and computationally investigate two different device architectures (Figure 1). The simplest type of NC-based solar cell consists of a metal- semiconductor-metal junction, the Schottky device (Figure 1a), since one of the metal-semiconductor interfaces forms a Schottky barrier. However, unlike a classical (unipolar) Schottky diode that creates a barrier for majority carriers, a NC-based Schottky solar cell is a bipolar device also allowing minority carrier injection (here, electrons into the p-type NC layer). Our device consists of a $100 \mathrm{~nm}$ thick lead sulfide (PbS) NC layer deposited on indium tin oxide (ITO) and topped with a $1 \mathrm{~nm}$ lithium fluoride $(\mathrm{LiF})$ and thick $(\sim 100 \mathrm{~nm})$ aluminum (Al) layer. For this work, we use PbS NCs that are 3 $\mathrm{nm}$ in diameter with ethanedithiol (EDT) as a cross-linking ligand. The properties of the PbS NCs and the device fabrication have been described in detail previously. ${ }^{23}$

A heterojunction device (Figure 1b) features an internal junction between the NC layer and another semiconducting material. The heterojunction devices used in this study consist of a $100 \mathrm{~nm}$ thick layer of zinc oxide $(\mathrm{ZnO}) \mathrm{NCs}$ deposited on ITO followed by a $350 \mathrm{~nm} \mathrm{PbS}$ layer with iodine surface termination. We consider the structure developed by Chuang et al., ${ }^{13}$ which includes an additional $\mathrm{PbS}$ layer cross-linked with EDT on top of the PbS:I layer. The device fabrication protocol used in this work used the solid-state ligand exchange reported previously ${ }^{24}$ and a slightly adapted recipe for $\mathrm{ZnO}$ nanoparticles (more $\mathrm{KOH}$ ), resulting in a highly doped n-type oxide. The device is completed with a top layer of gold. 
Simulation. An overview of the parameters used in the simulation are listed in Table 1, and the full parameter list can be found in Supporting Information, Table S1. We determine most of the input parameters independently from the experiment or calculation (Supporting Information). Below we discuss several important aspects related to the coarse grain parameterization of the $\mathrm{PbS}$ layers, the $\mathrm{ZnO}$ layer, and the interfaces that are relevant to the following discussions pertaining to device operation.

PbS NC Layer. The $\sim 3 \mathrm{~nm}$ PbS NCs used here are in the strong confinement regime, and the splitting between the highest occupied (lowest unoccupied) electronic state and the next lower (higher) state is $70 \mathrm{meV}(150 \mathrm{meV}) .^{26}$ Both energetic distances are significantly larger than $k T$, meaning that only the highest occupied (lowest unoccupied) electronic state will contribute to the valence band (VB) [conduction band (CB)] edges of the NC solid. We therefore take the effective density of states $N_{\mathrm{C}}=N_{\mathrm{V}}$ to be the density of NCs $N_{\mathrm{NC}}=10^{19} \mathrm{~cm}^{-3}$.

Electronic trap states have a significant impact on recombination and mobility in NC thin films. ${ }^{26}$ Trap states in $\mathrm{PbS}$ NC thin films have been shown to stem from both the oxidation or reduction of doped $\mathrm{NCs},{ }^{27}$ and the fusing two NCs together in a process known as NC dimerization. ${ }^{28}$ As thin-film fabrication of $\mathrm{PbS} \mathrm{NC}$ thin films is typically performed in air, we assume here a density, $N_{A}$ of p-type NCs resulting from exposure to oxygen. ${ }^{29}$ Based on measurements of the trap-state density, ${ }^{30}$ we assume that $1 \%$ of the NCs is of p-type (for a density $N_{\mathrm{A}} \sim 10^{17} \mathrm{~cm}^{-3}$ ), meaning that they have an additional hole, but their electronic structure is nearly identical to that of an intrinsic NC (Figure 2a). If this hole is released into the VB (i.e., the $\mathrm{NC}$ is reduced), the electronic levels of the then reduced p-type NC shift relative to the bands of the neighboring $\mathrm{NC}^{26}$ The highest occupied energy state of the reduced p-type NCs then become traps for holes in the NC film (Figure 2b). According to previously reported density functional theory (DFT) calculations, ${ }^{26}$ for a $3 \mathrm{~nm}$ diameter NC, this trap state will be at an energy $E_{\mathrm{T}, \mathrm{A}}$ of $0.25 \mathrm{eV}$ above the VB. Free carrier generation is therefore linked to the trap formation. This is taken into account in the simulations by doping the $\mathrm{PbS}$ layers using acceptors with a depth of $0.25 \mathrm{eV}$ and a concentration of $N_{\mathrm{A}} \sim 10^{17} \mathrm{~cm}^{-3}$. For simplicity, we begin by ignoring trap states arising from dimerization and treat this in the results and discussion section.

Recombination is a key parameter defining the efficiency of a solar cell. We apply the SRH recombination model, ${ }^{31}$ which describes the situation where an electron and hole recombine via a trap or defect state in the band gap of the semiconductor (e.g., an electron is captured and recombines with a hole in a trap). At the metallic contacts in the devices, surface recombination (i.e., the minority carrier current) is included. Recombination can also occur at the $\mathrm{ZnO} \mathrm{NC}$ and the $\mathrm{PbS} \mathrm{NC}$ interface; ${ }^{32-35}$ however, we assume here that this interface recombination is small relative to the trap-assisted bulk recombination (see the Supporting Information for further discussion). Additional recombination processes, such as radiative $\left(\sim n p / N_{\mathrm{NC}}{ }^{2}\right)$ or Auger Recombination $\left(\sim n^{2} p / \mathrm{N}_{\mathrm{NC}}{ }^{3}\right.$ or $\left.p^{2} n / N_{\mathrm{NC}}{ }^{3}\right)$, are not considered in our simulations. The rationale for the omission of both mechanisms is that they lead to substantially slower recombination than the SRH mechanism for the relatively low electron and hole densities $(n, p \ll$ $N_{\mathrm{NC}}$ ) relevant for illumination densities up to $1 \mathrm{sun}^{22}$
If the time needed for a charge carrier to find a recombination center in the SRH process is longer than the actual recombination process, we refer to this situation as the diffusion-limited regime. If the recombination process is longer than the time for carriers to find each other at the recombination center, we speak of the reaction-limited regime. In a study by Bozyigit et al., ${ }^{19}$ we showed that diffusion-limited recombination could explain the $I-V$ characteristics of both Schottky and heterojunction-based PbS NC devices over a large temperature range. We provide further discussion of the regimes in the Supporting Information.

To aid in the discussion of our simulations, we step through the equations relevant for SRH recombination following the presentation in ref 37 in the Supporting Information. In short, we can calculate the probability of a p-type NC having a hole, $f$, which is given by the hole capture $\left(p \nu_{\text {th.p }} \sigma_{\mathrm{p}}\right)$ plus the electron $\left(e_{n}\right)$ emission divided by capture and emission processes of both holes and electrons ${ }^{36}$

$$
f=\frac{p \nu_{\mathrm{th}, \mathrm{p}} \sigma_{\mathrm{p}}+\mathrm{e}_{\mathrm{n}}}{n \nu_{\mathrm{th}, \mathrm{n}} \sigma_{\mathrm{n}}+p \nu_{\mathrm{th}, \mathrm{p}} \sigma_{\mathrm{p}}+\mathrm{e}_{\mathrm{n}}+\mathrm{e}_{\mathrm{p}}}
$$

and the SRH recombination is given by

$$
R_{\mathrm{SRH}}=\frac{\nu_{\mathrm{th}, \mathrm{n}} \sigma_{\mathrm{n}} \nu_{\mathrm{th}, \mathrm{p}} \sigma_{\mathrm{p}}\left(n p-n_{i}^{2}\right)}{n \nu_{\mathrm{th}, \mathrm{n}} \sigma_{\mathrm{n}}+p \nu_{\mathrm{th}, \mathrm{p}} \sigma_{\mathrm{p}}+\mathrm{e}_{\mathrm{n}}+\mathrm{e}_{\mathrm{p}}} N_{\mathrm{A}}
$$

As we explain in the Supporting Information, we assume that the capture coefficients for electrons $\varphi_{\mathrm{n}}=\sigma_{\mathrm{n}} \nu_{\mathrm{th}, \mathrm{n}}$ and $\varphi_{\mathrm{p}}=\sigma_{\mathrm{p}}$ $\nu_{\text {th.p }}$ are equal, where $\sigma$ is the capture cross section and $\nu_{\text {th }}$ is thermal velocity of electrons and holes, respectively.

Lastly, we consider the electron and hole mobility. Charge transport in $\mathrm{PbS} \mathrm{NC}$ thin films occurs through phononmediated charge transfer between NCs, and we use DFTparameterized kinetic Monte Carlo simulations to determine an effective, temperature-dependent mobility for electrons, $\mu_{\mathrm{e}}(T)$, and holes, $\mu_{\mathrm{h}}\left(T, N_{\mathrm{T}}\right) .{ }^{26}$ Here, for all NC layers, we assume a NC size of $3 \mathrm{~nm}$ and an interparticle spacing of $6 \AA$, which leads to charge transfer times between two NCs of 21 ps. We use the DFT-based parameterization reported previously. ${ }^{26}$ For electrons, we find an effective mobility of $\mu_{\mathrm{e}}(300 \mathrm{~K})=1.65 \mathrm{~cm}^{2} / \mathrm{V}$ s. For holes, we determine $\mu_{\mathrm{h}}(300$ $\left.\mathrm{K}, 10^{17} \mathrm{~cm}^{-3}\right)=0.18 \mathrm{~cm}^{2} / \mathrm{V} \mathrm{s}$, which accounts for the presence of $10^{17} \mathrm{~cm}^{-3}$ reduced, p-type NCs that act as hole traps and significantly reduce average drift velocity of holes.

$\mathrm{ZnO}$ Layer. In the $\mathrm{NC}$-based $\mathrm{ZnO}$ layer, the electrons are the majority carriers (n-type semiconductors ${ }^{37}$ ) and contains no minority carrier in relevant densities. The electronic properties of $\mathrm{ZnO}$ vary greatly depending on the $\mathrm{ZnO}$ deposition method and post-deposition treatments. ${ }^{38} \mathrm{We}$ select electron and hole mobilities of 0.5 and $0.15 \mathrm{~cm}^{2} / \mathrm{V}$ $\mathrm{s},{ }^{39}$ based on literature values for nanostructured $\mathrm{ZnO}$ thin films. ${ }^{39-41}$ The $\mathrm{ZnO}$ layer has a large density of free electrons, where typical measured values range from $10^{18}$ to $10^{21}$ $\mathrm{cm}^{-3}$. ${ }^{39-41}$ These free electrons originate from donor states; however, little is understood about the origin of these donor states, ${ }^{42}$ making them difficult to use as a model.

The high $\mathrm{n}$-doping in $\mathrm{ZnO}$ is essential for the functionality of the heterojunction device; otherwise, a barrier exists at the $\mathrm{ZnO}-\mathrm{ITO}$ interface. If the donor density is too small $\left(<10^{18}\right.$ $\mathrm{cm}^{-3}$ ), an inverted diode is created across the entire $\mathrm{ZnO}$ layer at the $\mathrm{ZnO}-\mathrm{ITO}$ interface, impeding extraction of electrons at the ITO contact, opposing the current flow through the solar 
cell rendering the device non-operational (Supporting Information, Figure S4). For higher $n$-doping densities $\left(>10^{18} \mathrm{~cm}^{-3}\right)$, only a thin Schottky barrier remains at the $\mathrm{ZnO}-\mathrm{ITO}$ interface.

In the simulation, even this thin barrier impedes current flow across the $\mathrm{ZnO}-\mathrm{ITO}$ interface. We know that this is unphysical since previous studies ${ }^{13,24}$ on these types of solar cells, as well as our own experiments, demonstrate efficient electron extraction, meaning that current from $\mathrm{ZnO}$ to ITO is not impeded. This unphysical behavior of the simulation could have its origins either in the limitation of the simulation or the parameterization. Specifically, in the real device, tunneling through the thin barrier may occur that is not captured in the drift diffusion simulation. Additionally or alternatively, oxidized donors in the $\mathrm{ZnO}$ layer may be pinning the Fermi level of the $\mathrm{ZnO}$ somewhere between the $\mathrm{ZnO} \mathrm{CB}(-4.2 \mathrm{eV})$ and at a value closer to that of the ITO which is at $-4.9 \mathrm{eV}$, thus preventing the accumulation of free charge and barrier formation at the $\mathrm{ZnO}-\mathrm{ITO}$ interface. However, since we lack a model for the donor distribution in the $\mathrm{ZnO}$, we do not know where the Fermi level is pinned. We therefore model our $\mathrm{ZnO}$ layer as a trap-free semiconductor with a density of free electrons of $3 \times 10^{18} \mathrm{~cm}^{-3}$ and shift the ITO work function to $-4.6 \mathrm{eV}$, which prevents the formation of the injection barrier (Figure 2c). This simplification means that recombination dynamics in the $\mathrm{ZnO}$ is not treated; however, under all device operating conditions, the hole density in the $\mathrm{ZnO}$ is negligible.

Interfaces. Volk et al. $^{43}$ showed that at a $\mathrm{NC}-$ metal interface, a charge-transfer dipole layer is formed. Due to Fermi level equilibration, the NCs at the interface can either oxidize (or reduce). This dipole is located mostly across the first NC layer. As we cannot directly add a dipole to the simulation, we treat the metal and dipole as one element and shift the work function of the metal accordingly. ${ }^{22}$ We approximate the shift in Fermi level due to the surface dipole by $^{43}$

$$
\Delta E_{\mathrm{vac}}=\frac{|\mathbf{p}|}{\varepsilon_{0} \varepsilon_{\mathrm{r}} A_{\mathrm{NC}}}
$$

where $|\mathbf{p}|$ is the magnitude of the induced surface dipole. Assuming the charge forming the dipole is located in the center of the NCs in the first NC layer, ${ }^{43}$ the magnitude of the induced surface dipole is equivalent to a single charge times the NC center to metal contact distance, $|\mathbf{p}|=q \times\left(r_{\mathrm{NC}}+\right.$ $\left.r_{\mathrm{NC}-\mathrm{NC} \text { distance }}\right)=q \times 2.91 \mathrm{~nm} . A_{\mathrm{NC}}$ is effective substrate area occupied by a NC for the [100] facet of body-centered cubic (BCC) NC solid $\left(2 r_{\mathrm{NC}}+r_{\mathrm{NC}-\mathrm{NC} \text { distance }}\right)^{2}$ and has a value of $19.32 \mathrm{~nm}^{2}$ for EDT cross-linked $\mathrm{PbS}$. $\varepsilon_{\mathrm{r}}$ is the relative permittivity of $\mathrm{PbS}$ (Supporting Information, Table S1), and $\varepsilon_{0}$ is the vacuum permittivity. The shift in the Fermi level is therefore $\Delta E_{\mathrm{vac}}=195 \mathrm{meV}$.

Comparing Simulations to Experimental Device Characterization. As we have discussed, nearly all of the input parameters to the drift-diffusion simulations are independently taken from experiments or DFT basedcalculations. To confirm that the parameterization of the simulations can reproduce device operation, we perform a number of measurements on both Schottky and heterojunction devices.

Key performance metrics of a solar cell (short-circuit current, $J_{\mathrm{SC}}$, open-circuit voltage, $V_{\mathrm{OC}}$, and fill factor) can be obtained by measuring a current-voltage $(I-V)$ sweep under
AM1.5 illumination. In Figure 3a,b, we plot the measured light$I-V$ curves for the Schottky and heterojunction device,
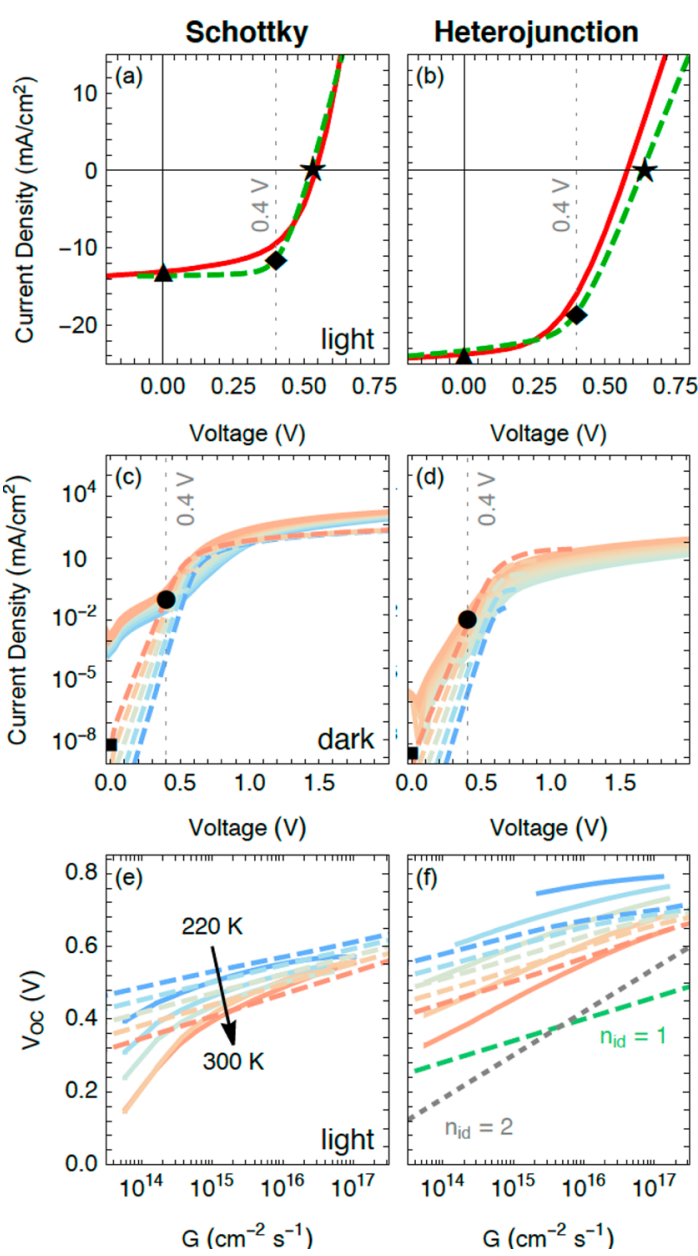

Figure 3. Measured (red solid line) and simulated (green dashed line) current-voltage sweep under AM1.5 illumination at $300 \mathrm{~K}$ of a Schottky (a) and a heterojunction (b) solar cell device. Measured (solid line) and simulated (dashed line) current-voltage sweeps in the dark for temperatures from 220 (blue) to $300 \mathrm{~K}$ (red) of a Schottky (c) and a heterojunction (d) solar cell device. Measured (solid line) and simulated (dashed) open-circuit voltage as a function of the generation rate and temperatures from 220 (blue) to $300 \mathrm{~K}$ (red) of a Schottky (e) and a heterojunction (f) solar cell device. The green dashed and gray dotted line mark the slopes for an ideality factor $n_{\text {id }}$ of 1 resp. 2 . In panels a-d, the triangles, diamonds, stars, squares, and circles mark the conditions where simulations are performed.

respectively. We utilize the light- $I-V$ curves to parameterize the two remaining "free" parameters in the simulations; the absorption cross section of the PbS NC thin film $\sigma_{\mathrm{A}}$, and the external series resistance of the devices $R_{\text {ser }}$ (which includes the sheet resistance of the ITO and interfacial resistances between the contact layers). Starting with previously reported values, ${ }^{19}$ both $\sigma_{\mathrm{A}}$ and $R_{\text {ser }}$ were fine tuned to optimize the agreement between the simulated and measured light- $I-V$ curves (Figure $3 \mathrm{a}, \mathrm{b})$. For the heterojunction device, the simulated $V_{\mathrm{OC}}$ is slightly higher than the measured value; however, it has been previously demonstrated that $V_{\mathrm{OC}}$ above $0.6 \mathrm{~V}$ can be achieved with this device architecture. ${ }^{24}$ Our slightly lower than expected $V_{\mathrm{OC}}$ could be due to the quality of the ligand exchange and environmental conditions during thin film 


\section{Schottky}

Column 1

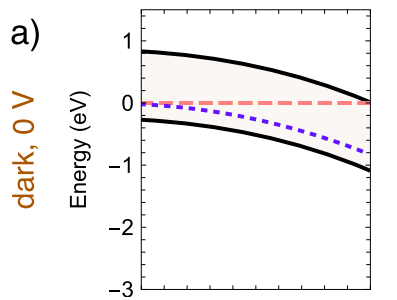

b)

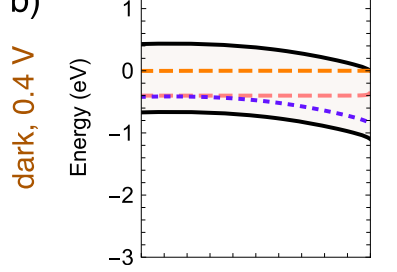

c)

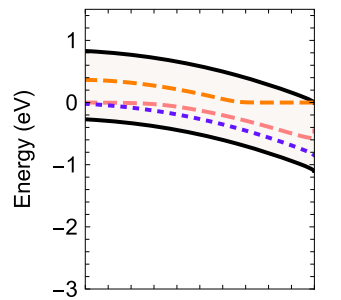

d)

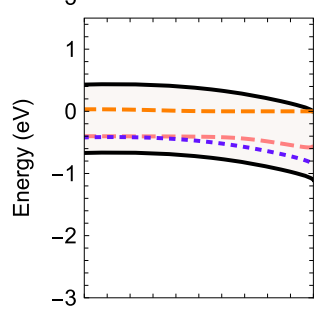

e)
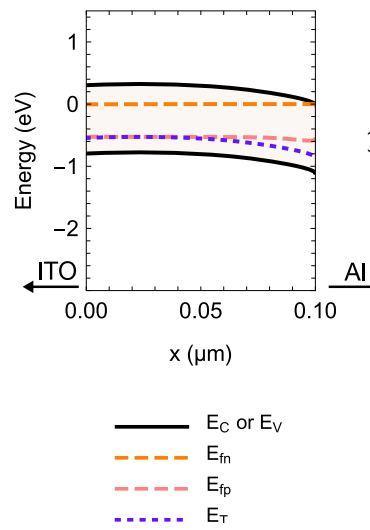

Column 2
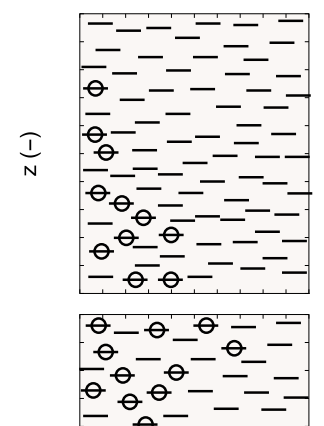

$\frac{I}{N}$

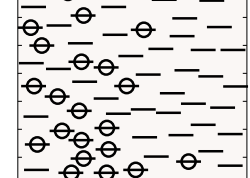

느- - -

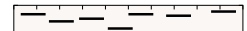

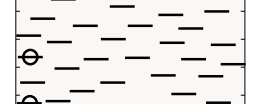

$\bar{\theta}--\overline{-}-\overline{-}$
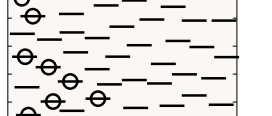

$\bar{\theta} \theta \bar{\theta}=\bar{\theta}-\overline{-}$
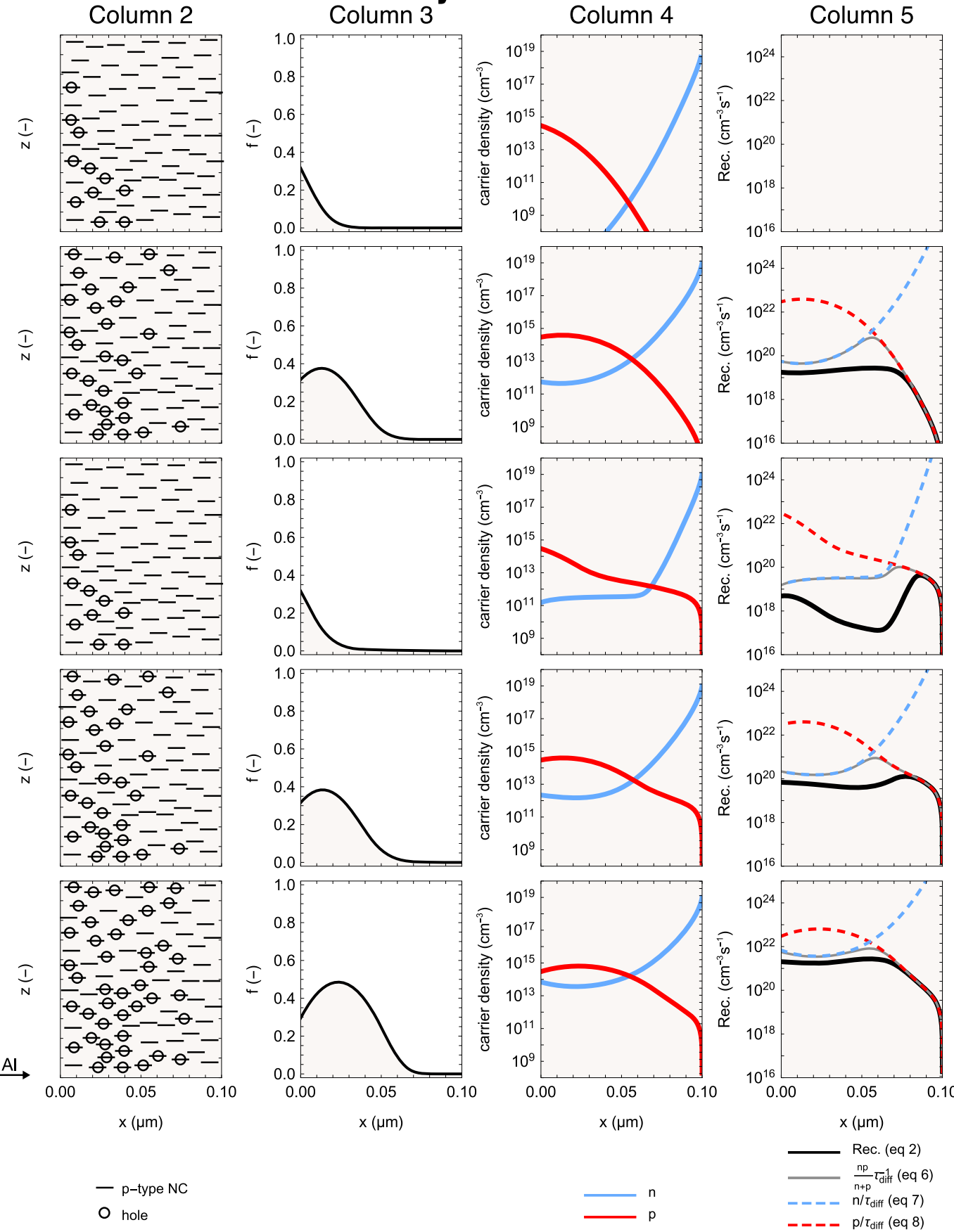

Figure 4. Band bending (column 1 ) across the PbS layer in the Schottky device is shown (a) in the dark at $0 \mathrm{~V}$ (square in Figure 3 ), (b) in the dark at $0.4 \mathrm{~V}$ (circle in Figure 3), (c) under 1 sun illumination at $0 \mathrm{~V}$ (triangle in Figure 3), (d) under 1 sun illumination at $0.4 \mathrm{~V}$ (diamond in Figure 3) and (e) under 1 sun illumination at $V_{\mathrm{OC}}$ (star in Figure 3). The level of acceptor defects is shown with the blue dotted line $\left(E_{\mathrm{T}}\right)$, and the quasiFermi levels $\left(E_{\mathrm{fn}}\right.$ and $\left.E_{\mathrm{fp}}\right)$ are shown with orange dashed lines. For each of these conditions, the additional columns present schematics showing where p-type NCs (represented by the "-") are occupied by a hole (represented by the "O") (column 2), the ratio of occupied traps (column 3), the charge carrier density (electrons shown with the blue line and holes shown with the red line) (column 4), and the effective recombination rate as given by eq 2 (black line), the recombination approximated by eq 6 (gray line), $n / \tau_{\text {diff }}$ (blue dashed line), and $p / \tau_{\text {diff }}$ (red dashed line) (column 5).

deposition, which influence the density of p-type NCs and dimer traps, which in turn will influence the recombination dynamics. However, the general agreement is a good first indication that our simulations can successfully reproduce the key performance metrics of the solar cell.
Temperature-dependent $I-V(I V T)$ sweeps in the dark have been previously used to study the charge-carrier transport, trapping, and recombination in NC-based solar cells. ${ }^{19}$ For both devices, we simulate the increase in current as a function of the voltage observed experimentally (Figure 3c,d). A plateau at $1 \mathrm{~V}$ is observed in both devices, which is due to the change 


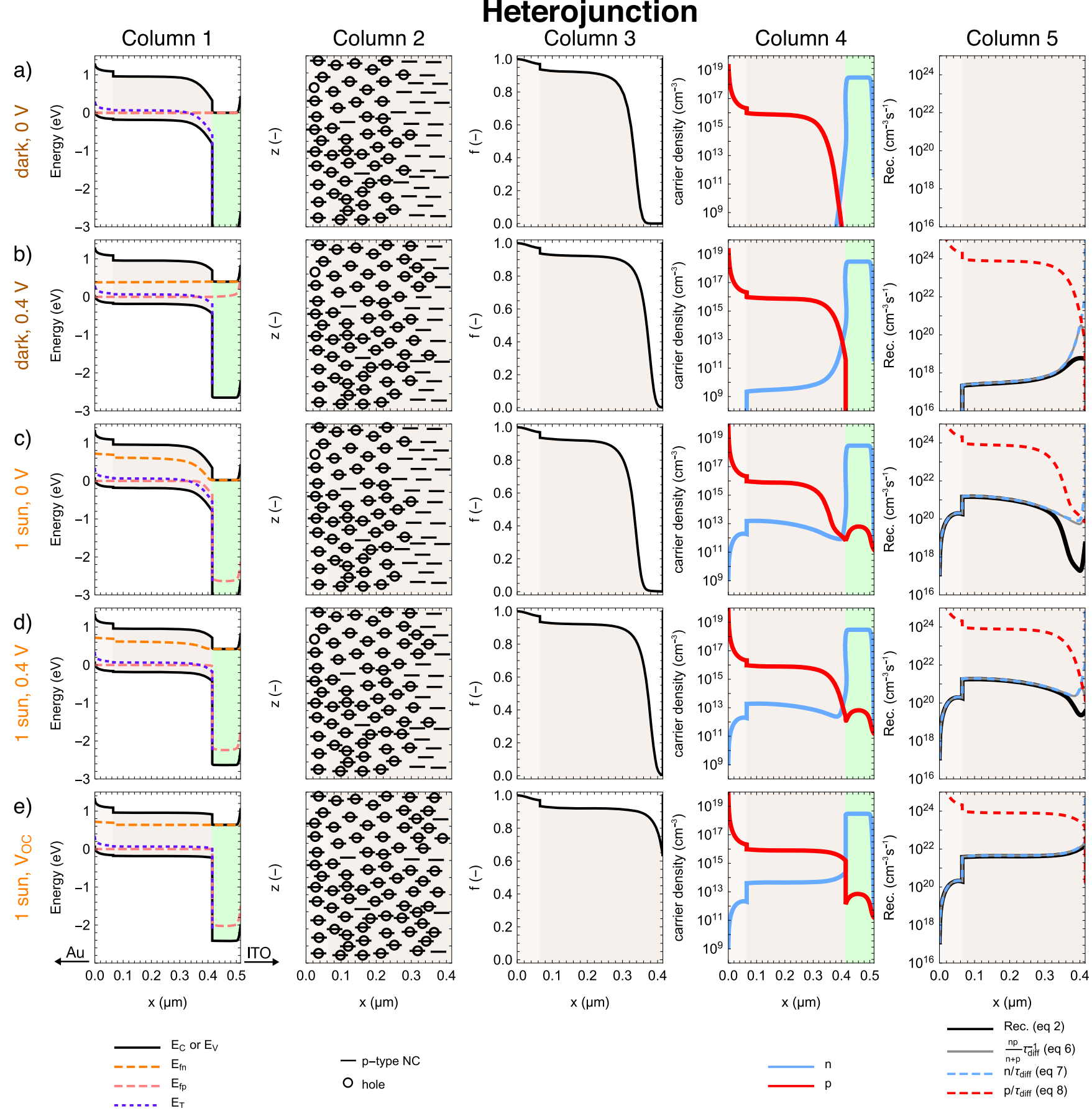

Figure 5. Band bending (column 1) across the PbS layers (PbS:EDT in light brown, $\mathrm{PbS}: \mathrm{I}$ in brown) and $\mathrm{ZnO}$ (green) for the heterojunction device is shown (a) in the dark at $0 \mathrm{~V}$ (square in Figure 3), (b) in the dark at $0.4 \mathrm{~V}$ (circle in Figure 3), (c) under 1 sun illumination at $0 \mathrm{~V}$ (triangle in Figure 3), (d) under 1 sun illumination at $0.4 \mathrm{~V}$ (diamond in Figure 3), and (e) under 1 sun illumination at $V_{\mathrm{OC}}$ (star in Figure 3). The level of acceptor defects is shown with the blue dotted line $\left(E_{\mathrm{T}}\right)$, and the quasi-Fermi levels $\left(E_{\mathrm{fn}}\right.$ and $\left.E_{\mathrm{fp}}\right)$ are shown with orange dashed lines. For each of these conditions, the additional columns present schematics showing where p-type NCs (represented by the "-") are occupied by a hole (represented by the "O") (column 2), the ratio of occupied traps (column 3), the charge carrier density (electrons shown with the blue line and holes shown with the red line) (column 4 ), and the effective recombination rate as given by eq 2 (black line), the recombination approximated by eq 6 (gray line), $n / \tau_{\text {diff }}$ (blue dashed line), and $p / \tau_{\text {diff }}$ (red dashed line) (column 5).

from a trap-assisted recombination regime at low voltage to a series resistance limited regime at higher voltages. The agreement in temperature dependence between the measurement and simulation shows that the assumptions are sufficient to capture the dominant temperature-dependent parameters. This is despite simplifications such as ignoring the temperature dependence of $\nu_{\text {th }}$ or the small differences in mobility expected in the PbS:I or the PbS:EDT. The discrepancy in current density for low voltage between the simulated and measured Schottky device (i.e., also seen by the different slopes at $0 \mathrm{~V}$ in Figure $3 a)$ is due to the absence of a finite shunt resistance in the simulated device, ignored for simplicity, which may be present in a real-device as a result of spatially inhomogeneous films or contaminates. ${ }^{11}$ 
Similarly, measurements of the open-circuit voltage as a function of temperature and generation rate, ${ }^{23} V_{\mathrm{OC}}(G, T)$, allow us to probe whether the recombination dynamics are accurately modeled. At $V_{\mathrm{OC}}$, no current is exiting the device, meaning that the recombination rate is equal to the generation rate. For both the Schottky and the heterojunction device, experiment and simulation agree and show that the $V_{\mathrm{OC}}$ increases with increasing generation rate (due to the higher number of photogenerated charge carriers) and decreases with increasing temperature (because $V_{\mathrm{OC}}=k T /\left(q \ln \left(n p / n_{i}^{2}\right)\right)$, and the equilibrium carrier concentration $n_{i}$ increases with increasing temperature so that the same amount of recombination is reached at a smaller quasi-Fermi level splitting) (Figure $3 \mathrm{e}, \mathrm{f})$. The slope of the $V_{\mathrm{OC}}$ versus generation rate (i.e., the ideality factor, see the Supporting Information) can provide insights into recombination mechanisms, and we discuss it in the results and discussion section below to further validate the simulation results. Ideality factors between 1 and 2 are typically indicative of SRH recombination, occurring in different regions of the device. In the Schottky device for low generation rates, $\left(G<10^{15} \mathrm{~cm}^{-3}\right)$, the experiment shows an ideality factor $>2$, whereas the simulation shows an ideality factor of 1 . An ideality factor $>2$ is consistent with the shunt resistance limiting performance, ${ }^{44}$ and, since we do not include a shunt resistance in the simulated device, it makes sense that this is not captured in the simulations. In the case of the heterojunction, we see that the simulations underestimate the $V_{\mathrm{OC}}$ at low temperatures and do not capture the experimentally observed behavior. This is likely a limitation of the parameterization since some parameters may have temperature dependence that we did not consider (e.g., mobility in the $\mathrm{ZnO}$, that would decrease for lower temperature which is equivalent to adding a series resistance). The trends in slope at higher generation rates are discussed below.

\section{RESULTS AND DISCUSSION}

Having confirmed that the simulations exhibit the key trends observed experimentally, we plot results of the simulation showing the energy bands within the PbS layer(s) (column 1), the occupation probability $f$ of a p-type NCs with a hole (columns 2 and 3 ), the density of electrons and holes (column 4 ), and the bulk recombination rate (column 5) in the Schottky (Figure 4) and heterojunction (Figure 5) devices for different operating conditions: (panel a) $0 \mathrm{~V}$ in the dark, (panel b) $0.4 \mathrm{~V}$ in the dark, (panel c) $0 \mathrm{~V}$ under 1 sun, (panel d) $0.4 \mathrm{~V}$ under 1 sun, and (panel e) $V_{\mathrm{OC}}$ under 1 sun. The 0.4 $\mathrm{V}$ forward bias was chosen as it is close to the maximal power point voltage of both devices (heterojunction $0.39 \mathrm{~V}$ and Schottky $0.38 \mathrm{~V}$ ). These simulations give us insights into the operation of NC-based solar cells.

To facilitate the discussion around the bulk recombination, in column 5, we also plot eq 2 under certain limits. If the intrinsic charge carrier density, ${ }^{36} n_{\mathrm{i}} \sim\left(N_{\mathrm{C}} N_{\mathrm{V}}\right)^{1 / 2} \times \mathrm{e}^{-E_{\mathrm{g}} / 2 k T}=$ $10^{9} \mathrm{~cm}^{-3}$, is much smaller than either $p$ or $n$, we can ignore this term in eq 2. Due to the large energetic distance of the trap states from the $\mathrm{CB}\left(E_{\mathrm{T}}-E_{\mathrm{C}}=0.9 \mathrm{eV}\right)$, the electron emission coefficient $e_{\mathrm{n}}$ is small $\left(\sim \nu_{\mathrm{th}, \mathrm{n}} \sigma_{\mathrm{n}} N_{\mathrm{c}} \mathrm{e}^{E_{\mathrm{C}}-E_{\mathrm{T}} / k T}=5 \times 10^{-5} \mathrm{~s}^{-1}\right.$ at $300 \mathrm{~K})$ and is therefore also ignored. The hole emission coefficient $e_{\mathrm{p}}$ is larger $\left(\sim \nu_{\mathrm{th}, \mathrm{p}} \sigma_{\mathrm{p}} N_{\mathrm{V}} \mathrm{e}^{E_{\mathrm{V}}-E_{\mathrm{T}} / k T}=6 \times 10^{5} \mathrm{~s}^{-1}\right.$ at $300 \mathrm{~K})$, but for most cases, $\nu_{\mathrm{th}, \mathrm{n}} \sigma_{\mathrm{n}} n$ or $\nu_{\mathrm{th}, \mathrm{p}} \sigma_{\mathrm{p}} p\left(\sim 10^{7}-10^{10} \mathrm{~s}^{-1}\right)$ is significantly larger than $e_{\mathrm{p}}$, so $e_{\mathrm{p}}$ can also be ignored. Therefore, eq 2 simplifies to

$$
R_{\mathrm{SRH}}=\frac{n p}{n+p} \sigma \nu_{\mathrm{th}} N_{\mathrm{A}}
$$

Defining a diffusion-limited $\mathrm{SRH}$ recombination time $\tau_{\mathrm{SRH}}$ constant

$$
\tau_{\mathrm{SRH}}=\frac{1}{\sigma \nu_{\mathrm{th}} N_{\mathrm{A}}}
$$

the recombination in the $\mathrm{PbS}$ layer can be expressed as

$$
R_{\mathrm{SRH}}=\frac{p n}{n+p} \frac{1}{\tau_{\mathrm{SRH}}}
$$

Equation 6 is shown with a gray dashed line column 5 .

Furthermore, if both $n$ and $p \gg N_{\mathrm{A}}$ and $p \ll n$ or $n \ll p$, eq 6 can be simplified to

$$
R_{p \gg n} \approx \frac{n}{\tau_{\text {diff }}}
$$

or

$$
R_{p \ll n} \approx \frac{p}{\tau_{\text {diff }}}
$$

Equations 7 and 8 are plotted in red and blue dashed lines in column 5 . We note that in column 5 , we are only considering the recombination that occurs in the $\mathrm{PbS}$ layer and not the surface recombination.

Schottky Device. The Schottky device at short-circuit conditions $(0 \mathrm{~V})$ shows significant band bending across the $\mathrm{PbS}$ NC layer (column 1, Figure 4a) due to the work function difference between the ITO and Al contacts. Most p-type NCs are missing holes (i.e., the PbS layer is mostly depleted) and is thus acting as trap states (column 2 and 3 ). To arrive at this condition, electrons (blue line in column 4) have entered the device from the aluminum contact and reduced the holes on the p-type NCs. At $0 \mathrm{~V}$ in the dark, the thermal generation and the recombination current are equal so no net recombination occurs (nothing is plotted in column 5).

When a positive voltage (here, $0.4 \mathrm{~V}$ ) is applied across the device (Figure 4b), electrons are injected from the $\mathrm{Al}$ contact, and holes are injected from the ITO contact. We see that the electron and hole densities (blue and red lines in column 4) are now distributed throughout the device. Because the application of a voltage flattens the bands, and injected holes can get trapped, there are fewer reduced p-type NCs (columns 2 and 3). The bulk recombination (black line in column 5) occurs predominately where there are p-type NCs occupied with a hole ( first $60 \mathrm{~nm}$ close to the ITO). Close to the $\mathrm{Al}$ contact, the electrons are majority carriers and as soon as a ptype NCs get occupied with a hole, an electron will recombine with the hole and reduce the NC again. Indeed, we are in the regime described by eq 8 where recombination is only limited by the number of holes (agreement between black line and red dashed line in column 5). Integrating the bulk recombination rate over the $\mathrm{PbS}$ layer thickness gives $1.5 \times 10^{14} \mathrm{~cm}^{-2} \mathrm{~s}^{-1}$. The injected current (both that measured and experimentally and in simulation, Figure 3c) is $0.12 \mathrm{~mA} \mathrm{~cm}^{-2}$, which corresponds to $7 \times 10^{14}$ electrons $\mathrm{cm}^{-2} \mathrm{~s}^{-1}$, meaning that most injected carriers recombine at the contacts (i.e., surface recombination dominates).

Under illumination, photons are absorbed, and electrons and holes are generated across the PbS layer (column 4). At shortcircuit condition $(0 \mathrm{~V})$ (Figure $4 \mathrm{c}$ ), the band bending is the same as in the dark (Figure 4a). While some photogenerated 


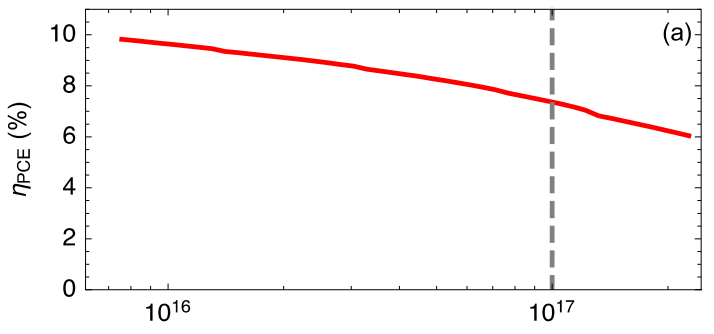

$N_{\mathrm{A}}\left(\mathrm{cm}^{-3}\right)$

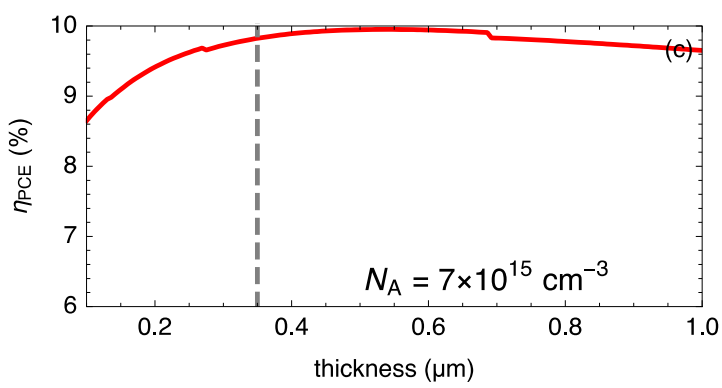

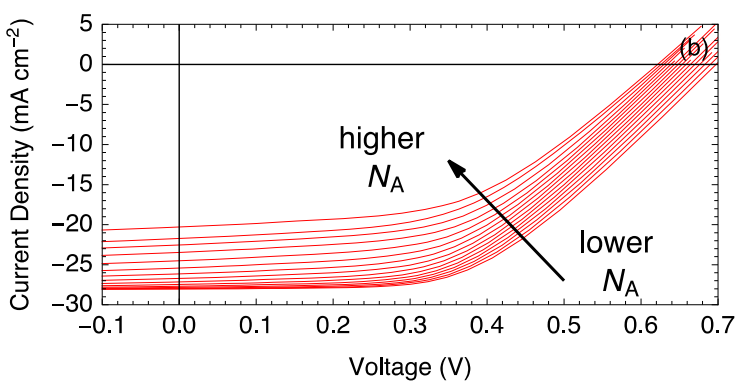

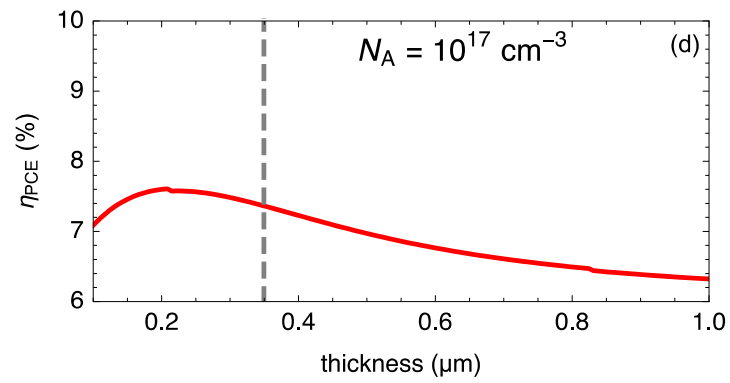

Figure 6. (a) Efficiency calculated as a function of the p-type NC density for a heterojunction device. Marked with a gray dashed line is the acceptor density of the solar cells used in this study. (b) Simulated current voltage sweeps under 1 sun illumination at $300 \mathrm{~K}$ of a heterojunction device at various acceptor densities (from $10^{14}$ to $2 \times 10^{17} \mathrm{~cm}^{-3}$ ). Efficiency as a function of PbS:I layer thickness for an acceptor density of $(\mathrm{c}) 10^{17}$ $\mathrm{cm}^{-3}$ (the same as in the real device) and (d) $7 \times 10^{15} \mathrm{~cm}^{-3}$. The gray dashed line indicates the layer thickness used in the experiments.

charge recombines, a short-circuit current of $J_{\mathrm{SC}}=13.6 \mathrm{~mA} /$ $\mathrm{cm}^{2}$ (i.e., electrons from aluminum) is extracted since the generation rate (i.e., the amount of charge carrier generated due to light $)$ is larger $\left(8.6 \times 10^{16} \mathrm{~cm}^{-2} \mathrm{~s}^{-1}\right)$ than the total recombination rate $\left(1.4 \times 10^{15} \mathrm{~cm}^{-2} \mathrm{~s}^{-1}\right)$. Surface recombination remains the dominant form of recombination $(1.3 \times$ $10^{15} \mathrm{~cm}^{-2} \mathrm{~s}^{-1}$ ). The highest rate of bulk recombination (black line in column 5, eq 2) occurs in the region near the aluminum contact. Here, we again have recombination in the space charge region, where the large number of majority electrons ( $p$ $\ll n)$ recombine with holes that can get trapped on reduced $\mathrm{p}$ doped NCs. The recombination depends only on the number of available holes (eq 8 , red dashed line).

As we increase the bias to $0.4 \mathrm{~V}$ (Figure $4 \mathrm{~d}$ ), the extracted current decreases $\left(11.6 \mathrm{~mA} / \mathrm{cm}^{2}\right)$ since the recombination increases $\left(\sim 0.25 \mathrm{~mA} \mathrm{~cm}{ }^{-2}\right)$. Here, the recombination in the neutral region of the device, where photogenerated electrons recombine with holes on p-type NCs that have not been depleted, is comparable to recombination in the space charge region. In the neutral region of the device, the recombination is limited by the number of electrons (blue dashed line, eq 7). We note that in the space-charge region, the assumption that the capture coefficients for electrons and holes are equivalent $\left(\varphi_{\mathrm{n}}=\varphi_{\mathrm{p}}=10^{-13} \mathrm{~cm}^{2}\right)$ may be less valid than that for the neutron region. For comparison, we simulate the case for 1 sun and $0.4 \mathrm{~V}$ where $\varphi_{\mathrm{p}} \gg \varphi_{\mathrm{n}}$ across the entire device and find that this would shift the region of maximum recombination into the space region (Supporting Information).

The flatter the bands, the higher the number of p-type NCs still occupied by holes. At $V_{\mathrm{OC}}$, we have almost full flat band condition, no charge is extracted (Figure 4e). Surface recombination still accounts for $80 \%$ of the total recombination $\left(8.6 \times 10^{16} \mathrm{~cm}^{-2} \mathrm{~s}^{-1}\right)$. The bulk recombination in the $\mathrm{PbS}$ $\left(1.6 \times 10^{16} \mathrm{~cm}^{-2} \mathrm{~s}^{-1}\right)$ accounts for $20 \%$ of the total and is dominated by the $\mathrm{SRH}$ in the neutral region.

In summary, the performance of a Schottky device is limited by the surface recombination (carriers going into wrong contact). This is consistent with the experimentally measured $V_{\mathrm{OC}}(G, T)$, as shown in Figure 3e, where we find an ideality factor $<1$ (Supporting Information), indicating that surface recombination is the limiting factor. ${ }^{45}$ As explained in previous work, ${ }^{22}$ this means that while some further improvements could be found by reducing the number of traps (i.e., p-doped NCs), the Schottky devices mostly are limited by the contacts (both in depletion width and surface recombination). Remedies can be the modification of the interface either by adding organic components ${ }^{46}$ or selecting electrodes with specific work functions to introduce, for example, beneficial interface dipoles. ${ }^{43}$

Heterojunction Devices. Figure 5 shows the same simulations as shown in Figure 4 but for heterojunction devices. The band diagrams in the first column highlight that the PbS:EDT layer works as an electron blocking layer and the $\mathrm{ZnO}$ as a hole blocking layer. Band bending (depletion region) in the $\mathrm{PbS}$ :I layer occurs predominately in the interface region with $\mathrm{ZnO}$, where a depletion region forms ( $\mathrm{p}$-doped NCs are reduced) due to electrons from the $\mathrm{ZnO}$ layer (columns 2 and 3). At $0 \mathrm{~V}$, in the dark (Figure 5a), the thickness of this depletion region is $\sim 100 \mathrm{~nm}$. While this is similar to the width of the depletion region in the Schottky device, heterojunction devices have a thicker PbS layer thickness (350 vs $100 \mathrm{~nm}$ ), so this depletion region is only a fraction of the $\mathrm{PbS}$ layer in the heterojunction, and, as we will see, is not critical to the device performance.

The relatively thin depletion region in the heterojunction device means that most p-doped NCs retain their holes (column 2 and 3 ) so that, for all operating conditions, the hole density is larger than the electron density (holes $\sim 10^{16} \mathrm{~cm}^{-3}$ and electrons $\sim 10^{9}$ to $10^{13} \mathrm{~cm}^{-3}$ ) throughout a large portion of the PbS:I NC layer. Since $p \gg n$, the bulk recombination rate in the $\mathrm{PbS}$ layer (black line in column 5 ) follows eq 7 , meaning that the recombination rate only depends on the number of available electrons. The overlap between the black line and blue dashed line for all operating conditions shows this to be 
the case. Only in a thin layer close to the $\mathrm{ZnO}$, where there are significant number of reduced NCs, does the bulk recombination rate deviate.

If a small bias $(0.4 \mathrm{~V})$ is applied to the device (Figure $5 \mathrm{~b}$ ), we inject a charge (current is $6.4 \times 10^{-3} \mathrm{~mA} \mathrm{~cm}^{-2}$ ). Due to the selective contacts, all injected charge recombines within the device (total recombination current $6.4 \times 10^{-3} \mathrm{~mA} \mathrm{~cm}^{-2}$ ). This is seen by the electron and hole densities (blue and red lines in column 4) going to zero in the PbS:EDT and $\mathrm{ZnO}$ layers, respectively. Adding light (Figure $5 \mathrm{c}-\mathrm{e}$ ), we photogenerate charge (generation rate $\left.=1.9 \times 10^{17} \mathrm{~cm}^{-2} \mathrm{~s}^{-1}\right)$. As a result of the electron and hole blocking (PbS:EDT and $\mathrm{ZnO}$ ), the charge that does not recombine in the PbS:I can be extracted.

In contrast to the Schottky device where surface recombination dominates, in the heterojunction device, at room temperature, SRH recombination accounts for the majority $(\sim 2 / 3)$ of the recombination. For example, at $0.4 \mathrm{~V}$ for the $30.6 \mathrm{~mA} \mathrm{~cm}^{-2}$ of photogenerated current, $6.0 \mathrm{~mA} \mathrm{~cm}{ }^{-2}$ is lost due to $\mathrm{SRH}$ recombination and $2.5 \mathrm{~mA} \mathrm{~cm}{ }^{-2}$ due to surface recombination, only $23.3 \mathrm{~mA} \mathrm{~cm}^{-2}$ can be extracted. This is consistent with the observation of experimental ideality factor near 1 , that only goes below 1 (i.e., indicating that the surface recombination is dominating) for low temperatures and high illumination intensities (Supporting Information).

Device Optimization. To improve the device performance in the heterojunction device, an obvious strategy is thus to reduce the number of hole traps. However, given the one-toone correspondence between the doping and trap density (i.e., the p-type NCs), decreasing the number of hole traps will consequently decrease the doping density and therefore reduce the strength and width of the built-in field. We assess whether eliminated doped NCs are nonetheless a viable strategy.

In Figure 6, we plot the power conversion efficiency, opencircuit voltage, and short-circuit current for a heterojunction device as function of p-type $\mathrm{NC}$ density $\left(N_{\mathrm{A}}\right)$ from $10^{15}$ (corresponding to 1 p-type NC per $10000 \mathrm{NCs}$ ) to $10^{18}$ $\mathrm{cm}^{-3}$ (corresponding to 1 per $10 \mathrm{NCs}$ ). The more doped the NCs, the worse the performance (Figure 6a), predominately due to the decrease in short-circuit current since more p-type NCs means higher recombination (Figure $6 \mathrm{~b}$ ). The $V_{\mathrm{OC}}$ is also weakly dependent on the density of p-type NCs as a reduction in the SRH recombination rate will also improve the $V_{\mathrm{OC}}$. We find that value for the $N_{\mathrm{A}}$ measured in $\mathrm{PbS} \mathrm{NC}$ solar cells of $10^{17} \mathrm{~cm}^{-3}$ (gray dashed line in Figure 6a) lies in a portion of the plot with a steep slope. This means that small variations in $N_{\mathrm{A}}$ can lead to significant changes in the performance, which explains why small changes to the system that can influence the NC doping (e.g., storage time of NCs, condition during thin film deposition, quality of chemicals and precursor) and strongly impact performance. While it appears that reducing the number of recombination centers $N_{\mathrm{A}}$ improves the performance dramatically, in reality other forms of recombination (e.g., band-to-band or Auger ${ }^{47}$ would begin to dominate (Supporting Information).

For an acceptor density of $10^{17} \mathrm{~cm}^{-3}$, increasing the thickness further would reduce the overall efficiency (Figure $6 \mathrm{~d})$. Decreasing the p-type NC density (e.g., $7 \times 10^{15} \mathrm{~cm}^{-3}$ in Figure 6c) would allow more charge carriers to be extracted before they recombine, enabling an increase in the active layer thickness. This allows more absorption and therefore increase the overall efficiency of the device.
While reduction of a p-type NC creates a trap for holes and acts as a recombination center, we also know that fusing NCs into dimers also creates deep trap states. ${ }^{28}$ In the Supporting Information, we propose how to model recombination in the dimers and find that the dimers alone cannot account for the recombination dynamics observed experimentally in these devices.

\section{CONCLUSIONS}

In conclusion, device level simulations of PbS NC-based solar cells enable us to identify the origin of performance limitations and to assess where further development efforts should be focused. We identify three aspects to consider.

First, in terms of NC materials, we show that the p-doping and electronic defects (e.g., dimer traps) both have a negative impact on the performance. This reinforces the importance of synthesis, washing procedures, and deposition conditions on the final device performance.

Second, electron and hole transport and blocking layers limit the performance and should be improved. Both the PbS:EDT and $\mathrm{ZnO}$ play an important role in blocking electrons and holes from recombining at the contacts. An important learning from this work came from the parameterization of the $\mathrm{ZnO}$ and $\mathrm{ZnO}-\mathrm{ITO}$ contact. We observe that it is crucial to have highly defective $\mathrm{ZnO}$ that pins the work function of the ITO. The high electronic density is not crucial to the solar cell but rather helps fix the $\mathrm{ZnO}-\mathrm{ITO}$ interface. This can be further improved, for example, by magnesium doping ${ }^{48}$ or indium doping of $\mathrm{ZnO}^{49} \mathrm{On}$ the hole transport (electron blocking) layer, a number of recent studies are exploring different organic molecules ${ }^{50}$ and polymers. ${ }^{51}$

Third, interfaces with contacts require consideration. Due to the dipole formation on the $\mathrm{NC}$-contact interface, the contact work-functions must be selected with awareness of the NC electronic structure. ${ }^{43}$ Alternately, the chemical modification to the interface or the surface of the NC can mediate this effect, as shown in recent work. ${ }^{52}$

While this work has been carried out for PbS NC solar cells, we hope the approach involving independent parameterization of drift-diffusion simulations will inspire analogous work on other NC solar cells. For example, parameterization and simulation of NC perovskite solar cells ${ }^{53,54}$ could hopefully accelerate targeted development of techniques for NC preparation and assembly in devices. Size-dependent charge separation and recombination have been demonstrated, ${ }^{55}$ and optimization of NC preparation can improve carrier charge mobility and device efficiency. ${ }^{56}$ Such results, complemented by other design choices based on an understanding of charge distributions and recombination dynamics obtained through bottom-up parameterized simulations, can be used to further push the limits of the NC solar cell performance.

\section{ASSOCIATED CONTENT}

\section{Supporting Information}

The Supporting Information is available free of charge at https://pubs.acs.org/doi/10.1021/acsaelm.1c00787.

Complete list of all parameters used in the simulations and justifications for parameter selection, discussion of device ideality factors, further descriptions and analysis of charge generation, capture, recombination in the devices (PDF) 


\section{AUTHOR INFORMATION}

\section{Corresponding Author}

Vanessa Wood - Department of Information Technology and Electrical Engineering, ETH Zürich, 8092 Zürich,

Switzerland; 이이.org/0000-0001-6435-0227;

Email: vwood@ethz.ch

\section{Authors}

Weyde M. M. Lin - Department of Information Technology and Electrical Engineering, ETH Zürich, 8092 Zürich, Switzerland; 이이이.org/0000-0002-7572-499X

Nuri Yazdani - Department of Information Technology and Electrical Engineering, ETH Zürich, 8092 Zürich, Switzerland; () orcid.org/0000-0001-6593-7601

Olesya Yarema - Department of Information Technology and Electrical Engineering, ETH Zürich, 8092 Zürich, Switzerland

Maksym Yarema - Department of Information Technology and Electrical Engineering, ETH Zürich, 8092 Zürich, Switzerland; (ㅇ) orcid.org/0000-0002-2006-2466

Mengxia Liu - Department of Electrical and Computer Engineering, University of Toronto, Toronto M5S 3G4, Canada; 10 orcid.org/0000-0002-1676-705X

Edward H. Sargent - Department of Electrical and Computer Engineering, University of Toronto, Toronto MSS 3G4, Canada; () orcid.org/0000-0003-0396-6495

Thomas Kirchartz - IEK5-Photovoltaik, Forschungszentrum Jülich, 52425 Jülich, Germany; Faculty of Engineering and CENIDE, University of Duisburg-Essen, 47057 Duisburg,

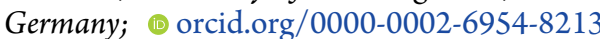

Complete contact information is available at: https://pubs.acs.org/10.1021/acsaelm.1c00787

\section{Notes}

The authors declare no competing financial interest.

\section{ACKNOWLEDGMENTS}

The authors acknowledge funding from an ETH Research Grant (ETH-42 12-2) (W.M.M.L., N.Y., and V.W.), the Swiss National Science Foundation (O.Y., M.Y., and V.W.), the Natural Sciences and Engineering Research Council of Canada (NSERC) through the Discovery program, and the support of the Canada Research Chairs program (M.L. and E.H.S.). The authors thank Prof. Marc Burgelman for his assistance with SCAPs and Prof. Uwe Kortshagen, Zachary Robinson, and Chengjian Zhang from the University of Minnesota for helpful discussion regarding $\mathrm{ZnO}$ nanoparticles.

\section{REFERENCES}

(1) Hines, M. A.; Scholes, G. D. Colloidal PbS Nanocrystals with Size-Tunable Near-Infrared Emission: Observation of Post-Synthesis Self-Narrowing of the Particle Size Distribution. Adv. Mater. 2003, 15, 1844-1849.

(2) Zheng, S.; Chen, J.; Johansson, E. M. J.; Zhang, X. PbS Colloidal Quantum Dot Inks for Infrared Solar Cells. iScience 2020, 23, 101753.

(3) Gur, I.; Fromer, N. A.; Geier, M. L.; Alivisatos, A. P. Air-Stable All-Inorganic Nanocrystal Solar Cells Processed from Solution. Science 2005, 310, 462-465.

(4) Hao, M.; Bai, Y.; Zeiske, S.; Ren, L.; Liu, J.; Yuan, Y.; Zarrabi, N.; Cheng, N.; Ghasemi, M.; Chen, P.; Lyu, M.; He, D.; Yun, J.-H.; Du, Y.; Wang, Y.; Ding, S.; Armin, A.; Meredith, P.; Liu, G.; Cheng, H.-M.; Wang, L. Ligand-Assisted Cation-Exchange Engineering for High-Efficiency Colloidal Cs1-xFAxPbI3 Quantum Dot Solar Cells with Reduced Phase Segregation. Nat. Energy 2020, 5, 79-88.
(5) Lin, W. M. M.; Yarema, M.; Liu, M.; Sargent, E.; Wood, V. Nanocrystal Quantum Dot Devices: How the Lead Sulfide (PbS) System Teaches Us the Importance of Surfaces. Chimia 2021, 75, 398.

(6) Klem, E. J. D.; Shukla, H.; Hinds, S.; MacNeil, D. D.; Levina, L.; Sargent, E. H. Impact of Dithiol Treatment and Air Annealing on the Conductivity, Mobility, and Hole Density in PbS Colloidal Quantum Dot Solids. Appl. Phys. Lett. 2008, 92, 212105.

(7) Ip, A. H.; Thon, S. M.; Hoogland, S.; Voznyy, O.; Zhitomirsky, D.; Debnath, R.; Levina, L.; Rollny, L. R.; Carey, G. H.; Fischer, A.; Kemp, K. W.; Kramer, I. J.; Ning, Z.; Labelle, A. J.; Chou, K. W.; Amassian, A.; Sargent, E. H. Hybrid Passivated Colloidal Quantum Dot Solids. Nat. Nanotechnol. 2012, 7, 577-582.

(8) Kahmann, S.; Loi, M. A. Trap States in Lead Chalcogenide Colloidal Quantum Dots-Origin, Impact, and Remedies. Appl. Phys. Rev. 2020, 7, 041305.

(9) Kahmann, S.; Sytnyk, M.; Schrenker, N.; Matt, G. J.; Spiecker, E.; Heiss, W.; Brabec, C. J.; Loi, M. A. Revealing Trap States in Lead Sulphide Colloidal Quantum Dots by Photoinduced Absorption Spectroscopy. Adv. Electron. Mater. 2018, 4, 1700348.

(10) Cao, Y.; Stavrinadis, A.; Lasanta, T.; So, D.; Konstantatos, G. The Role of Surface Passivation for Efficient and Photostable $\mathrm{PbS}$ Quantum Dot Solar Cells. Nat. Energy 2016, 1, 16035.

(11) Yoon, W.; Boercker, J. E.; Lumb, M. P.; Placencia, D.; Foos, E. E.; Tischler, J. G. Enhanced Open-Circuit Voltage of PbS Nanocrystal Quantum Dot Solar Cells. Sci. Rep. 2013, 3, 2225.

(12) Hu, L.; Lei, Q.; Guan, X.; Patterson, R.; Yuan, J.; Lin, C.-H.; Kim, J.; Geng, X.; Younis, A.; Wu, X.; Liu, X.; Wan, T.; Chu, D.; Wu, T.; Huang, S. Optimizing Surface Chemistry of $\mathrm{PbS}$ Colloidal Quantum Dot for Highly Efficient and Stable Solar Cells via Chemical Binding. Adv. Sci. 2021, 8, 2003138.

(13) Chuang, C.-H. M.; Brown, P. R.; Bulović, V.; Bawendi, M. G. Improved Performance and Stability in Quantum Dot Solar Cells through Band Alignment Engineering. Nat. Mater. 2014, 13, 796801.

(14) Hu, L.; Zhang, Z.; Patterson, R. J.; Hu, Y.; Chen, W.; Chen, C.; Li, D.; Hu, C.; Ge, C.; Chen, Z.; Yuan, L.; Yan, C.; Song, N.; Teh, Z. L.; Conibeer, G. J.; Tang, J.; Huang, S. Achieving High-Performance $\mathrm{PbS}$ Quantum Dot Solar Cells by Improving Hole Extraction through Ag Doping. Nano Energy 2018, 46, 212-219.

(15) Choi, M.-J.; García de Arquer, F. P.; Proppe, A. H.; Seifitokaldani, A.; Choi, J.; Kim, J.; Baek, S.-W.; Liu, M.; Sun, B.; Biondi, M.; Scheffel, B.; Walters, G.; Nam, D.-H.; Jo, J. W.; Ouellette, O.; Voznyy, O.; Hoogland, S.; Kelley, S. O.; Jung, Y. S.; Sargent, E. H. Cascade Surface Modification of Colloidal Quantum Dot Inks Enables Efficient Bulk Homojunction Photovoltaics. Nat. Commun. 2020, 11, 103.

(16) Ning, Z.; Zhitomirsky, D.; Adinolfi, V.; Sutherland, B.; Xu, J.; Voznyy, O.; Maraghechi, P.; Lan, X.; Hoogland, S.; Ren, Y.; Sargent, E. H. Graded Doping for Enhanced Colloidal Quantum Dot Photovoltaics. Adv. Mater. 2013, 25, 1719-1723.

(17) Fabregat-Santiago, F.; Garcia-Belmonte, G.; Mora-Seró, I.; Bisquert, J. Characterization of Nanostructured Hybrid and Organic Solar Cells by Impedance Spectroscopy. Phys. Chem. Chem. Phys. 2011, 13, 9083

(18) Kirchartz, T.; Ding, K.; Rau, U. Fundamental Electrical Characterization of Thin-Film Solar Cells. Advanced Characterization Techniques for Thin Film Solar Cells; Wiley-VCH Verlag GmbH \& Co. KGaA: Weinheim, Germany, 2011; pp 33-60.

(19) Bozyigit, D.; Lin, W. M. M.; Yazdani, N.; Yarema, O.; Wood, V. A Quantitative Model for Charge Carrier Transport, Trapping and Recombination in Nanocrystal-Based Solar Cells. Nat. Commun. 2015, 6, 6180.

(20) Hu, L.; Liu, M.; Mandelis, A.; Melnikov, A.; Sargent, E. H. Colloidal Quantum Dot Solar Cell Power Conversion Efficiency Optimization Using Analysis of Current-Voltage Characteristics and Electrode Contact Imaging by Lock-in Carrierography. Prog. Photovoltaics Res. Appl. 2017, 25, 1034-1050. 
(21) Kirchartz, T.; Nelson, J. Device Modelling of Organic Bulk Heterojunction Solar Cells. Multiscale Modelling of Organic And Hybrid Photovoltaics; Springer, 2013; pp 279-324.

(22) Lin, W. M. M.; Yazdani, N.; Yarema, O.; Volk, S.; Yarema, M.; Kirchartz, T.; Wood, V. Simulating Nanocrystal-Based Solar Cells: A Lead Sulfide Case Study. J. Chem. Phys. 2019, 151, 241104.

(23) Lin, W. M. M.; Bozyigit, D.; Yarema, O.; Wood, V. Transient Photovoltage Measurements in Nanocrystal-Based Solar Cells. J. Phys. Chem. C 2016, 120, 12900-12908.

(24) Liu, M.; Voznyy, O.; Sabatini, R.; García de Arquer, F. P.; Munir, R.; Balawi, A. H.; Lan, X.; Fan, F.; Walters, G.; Kirmani, A. R.; Hoogland, S.; Laquai, F.; Amassian, A.; Sargent, E. H. Hybrid Organic-Inorganic Inks Flatten the Energy Landscape in Colloidal Quantum Dot Solids. Nat. Mater. 2017, 16, 258-263.

(25) Peng, H.; Liu, X.; Tang, W.; Ma, R. Facile Synthesis and Characterization of $\mathrm{ZnO}$ Nanoparticles Grown on Halloysite Nanotubes for Enhanced Photocatalytic Properties. Sci. Rep. 2017, 7, 2250.

(26) Yazdani, N.; Andermatt, S.; Yarema, M.; Farto, V.; BaniHashemian, M. H.; Volk, S.; Lin, W. M. M.; Yarema, O.; Luisier, M.; Wood, V. Charge Transport in Semiconductors Assembled from Nanocrystal Quantum Dots. Nat. Commun. 2020, 11, 2852.

(27) Volk, S.; Yazdani, N.; Yarema, O.; Yarema, M.; Wood, V. Dopants and Traps in Nanocrystal-Based Semiconductor Thin Films: Origins and Measurement of Electronic Midgap States. ACS Appl. Electron. Mater. 2020, 2, 398-404.

(28) Gilmore, R. H.; Liu, Y.; Shcherbakov-Wu, W.; Dahod, N. S.; Lee, E. M. Y.; Weidman, M. C.; Li, H.; Jean, J.; Bulović, V.; Willard, A. P.; Grossman, J. C.; Tisdale, W. A. Epitaxial Dimers and AugerAssisted Detrapping in PbS Quantum Dot Solids. Matter 2019, 1, 250-265.

(29) Voznyy, O.; Zhitomirsky, D.; Stadler, P.; Ning, Z.; Hoogland, S.; Sargent, E. H. A Charge-Orbital Balance Picture of Doping in Colloidal Quantum Dot Solids. ACS Nano 2012, 6, 8448-8455.

(30) Bozyigit, D.; Volk, S.; Yarema, O.; Wood, V. Quantification of Deep Traps in Nanocrystal Solids, Their Electronic Properties, and Their Influence on Device Behavior. Nano Lett. 2013, 13, 5284-5288.

(31) Sze, S. M.; Ng, K. K. Physics of Semiconductor Devices; John Wiley \& Sons, 2006.

(32) Zhang, X.; Johansson, E. M. J. Reduction of Charge Recombination in PbS Colloidal Quantum Dot Solar Cells at the Quantum Dot/ZnO Interface by Inserting a MgZnO Buffer Layer. J. Mater. Chem. A 2017, 5, 303-310.

(33) Ding, C.; Zhang, Y.; Liu, F.; Kitabatake, Y.; Hayase, S.; Toyoda, T.; Wang, R.; Yoshino, K.; Minemoto, T.; Shen, Q. Understanding Charge Transfer and Recombination by Interface Engineering for Improving the Efficiency of PbS Quantum Dot Solar Cells. Nanoscale Horiz. 2018, 3, 417-429.

(34) Pradhan, S.; Stavrinadis, A.; Gupta, S.; Konstantatos, G. Reducing Interface Recombination through Mixed Nanocrystal Interlayers in $\mathrm{PbS}$ Quantum Dot Solar Cells. ACS Appl. Mater. Interfaces 2017, 9, 27390-27395.

(35) Cheng, J. J.; Chuang, C.-H. M.; Hentz, O.; Rekemeyer, P. H.; Bawendi, M. G.; Gradečak, S. Dimension- and Surface-Tailored ZnO Nanowires Enhance Charge Collection in Quantum Dot Photovoltaic Devices. ACS Appl. Energy Mater. 2018, 1, 1815-1822.

(36) Pieters, B. E.; Decock, K.; Burgelman, M.; Stangl, R.; Kirchartz, T. One-Dimensional Electro-Optical Simulations of Thin-Film Solar Cells. In Advanced Characterization Techniques for Thin Film Solar Cells; Abou-Ras, D., Kirchartz, T., Rau, U., Eds.; Wiley-VCH Verlag GmbH \& Co. KGaA: Weinheim, Germany, 2016; pp 633-657.

(37) Özgür, Ü.; Alivov, Y. I.; Liu, C.; Teke, A.; Reshchikov, M. A.; Doğan, S.; Avrutin, V.; Cho, S.-J.; Morkoç, H. A Comprehensive Review of $\mathrm{ZnO}$ Materials and Devices. J. Appl. Phys. 2005, 98, 041301.

(38) Djurišíć, A. B.; Chen, X.; Leung, Y. H.; Man Ching Ng, A. ZnO Nanostructures: Growth, Properties and Applications. J. Mater. Chem. 2012, 22, 6526 .
(39) Greenberg, B. L.; Robinson, Z. L.; Reich, K. V.; Gorynski, C.; Voigt, B. N.; Francis, L. F.; Shklovskii, B. I.; Aydil, E. S.; Kortshagen, U. R. ZnO Nanocrystal Networks Near the Insulator-Metal Transition: Tuning Contact Radius and Electron Density with Intense Pulsed Light. Nano Lett. 2017, 17, 4634-4642.

(40) Greenberg, B. L.; Ganguly, S.; Held, J. T.; Kramer, N. J.; Mkhoyan, K. A.; Aydil, E. S.; Kortshagen, U. R. NonequilibriumPlasma-Synthesized $\mathrm{ZnO}$ Nanocrystals with Plasmon Resonance Tunable via Al Doping and Quantum Confinement. Nano Lett. 2015, 15, 8162-8169.

(41) Han, W.; Kim, J.; Park, H.-H. Control of Electrical Conductivity of Highly Stacked Zinc Oxide Nanocrystals by Ultraviolet Treatment. Sci. Rep. 2019, 9, 6244.

(42) Penfold, T. J.; Szlachetko, J.; Santomauro, F. G.; Britz, A.; Gawelda, W.; Doumy, G.; March, A. M.; Southworth, S. H.; Rittmann, J.; Abela, R.; Chergui, M.; Milne, C. J. Revealing Hole Trapping in Zinc Oxide Nanoparticles by Time-Resolved X-Ray Spectroscopy. Nat. Commun. 2018, 9, 478.

(43) Volk, S.; Yazdani, N.; Yarema, O.; Yarema, M.; Bozyigit, D.; Wood, V. In Situ Measurement and Control of the Fermi Level in Colloidal Nanocrystal Thin Films during Their Fabrication. J. Phys. Chem. Lett. 2018, 9, 7165.

(44) Bunea, G.; Wilson, K.; Meydbray, Y.; Campbell, M.; De Ceuster, D. Low Light Performance of Mono-Crystalline Silicon Solar Cells. IEEE 4th World Conference on Photovoltaic Energy Conference; IEEE, 2006; pp 1312-1314.

(45) Kirchartz, T.; Deledalle, F.; Tuladhar, P. S.; Durrant, J. R.; Nelson, J. On the Differences between Dark and Light Ideality Factor in Polymer:Fullerene Solar Cells. J. Phys. Chem. Lett. 2013, 4, 23712376.

(46) Asyuda, A.; Gärtner, M.; Wan, X.; Burkhart, I.; Saßmannshausen, T.; Terfort, A.; Zharnikov, M. Self-Assembled Monolayers with Embedded Dipole Moments for Work Function Engineering of Oxide Substrates. J. Phys. Chem. C 2020, 124, 87758785.

(47) Istrate, E.; Hoogland, S.; Sukhovatkin, V.; Levina, L.; Myrskog, S.; Smith, P. W. E.; Sargent, E. H. Carrier Relaxation Dynamics in Lead Sulfide Colloidal Quantum Dots. J. Phys. Chem. B 2008, 112, $2757-2760$.

(48) Xing, M.; Wei, Y.; Wang, D.; Shen, Q.; Wang, R. Mg-Doped $\mathrm{ZnO}$ Layer to Enhance Electron Transporting for PbS Quantum Dot Solar Cells. Curr. Appl. Phys. 2021, 21, 14-19.

(49) Liu, M.; de Arquer, F. P. G.; Li, Y.; Lan, X.; Kim, G.-H.; Voznyy, O.; Jagadamma, L. K.; Abbas, A. S.; Hoogland, S.; Lu, Z.; Kim, J. Y.; Amassian, A.; Sargent, E. H. Double-Sided Junctions Enable High-Performance Colloidal-Quantum-Dot Photovoltaics. Adv. Mater. 2016, 28, 4142-4148.

(50) Chiu, A.; Rong, E.; Bambini, C.; Lin, Y.; Lu, C.; Thon, S. M. Sulfur-Infused Hole Transport Materials to Overcome PerformanceLimiting Transport in Colloidal Quantum Dot Solar Cells. ACS Energy Lett. 2020, 5, 2897-2904.

(51) Al Mubarok, M.; Wibowo, F. T. A.; Aqoma, H.; Vamsi Krishna, N.; Lee, W.; Ryu, D. Y.; Cho, S.; Jung, I. H.; Jang, S.-Y. PbS-Based Quantum Dot Solar Cells with Engineered $\pi$-Conjugated Polymers Achieve 13\% Efficiency. ACS Energy Lett. 2020, 5, 3452-3460.

(52) Mubarok, M. A.; Aqoma, H.; Wibowo, F. T. A.; Lee, W.; Kim, H. M.; Ryu, D. Y.; Jeon, J.-W.; Jang, S.-Y. Molecular Engineering in Hole Transport П-Conjugated Polymers to Enable High Efficiency Colloidal Quantum Dot Solar Cells. Adv. Energy Mater. 2020, 10, 1902933.

(53) Que, M.; Zhu, L.; Guo, Y.; Que, W.; Yun, S. Toward Perovskite Nanocrystalline Solar Cells: Progress and Potential. J. Mater. Chem. C 2020, 8, 5321-5334.

(54) Duan, L.; Hu, L.; Guan, X.; Lin, C.-H.; Chu, D.; Huang, S.; Liu, X.; Yuan, J.; Wu, T. Quantum Dots for Photovoltaics: A Tale of Two Materials. Adv. Energy Mater. 2021, 11, 2100354.

(55) Shang, Q.; Kaledin, A. L.; Li, Q.; Lian, T. Size Dependent Charge Separation and Recombination in CsPbI 3 Perovskite Quantum Dots. J. Chem. Phys. 2019, 151, 074705. 
(56) Yuan, J.; Bi, C.; Wang, S.; Guo, R.; Shen, T.; Zhang, L.; Tian, J. Spray-Coated Colloidal Perovskite Quantum Dot Films for Highly Efficient Solar Cells. Adv. Funct. Mater. 2019, 29, 1906615. 